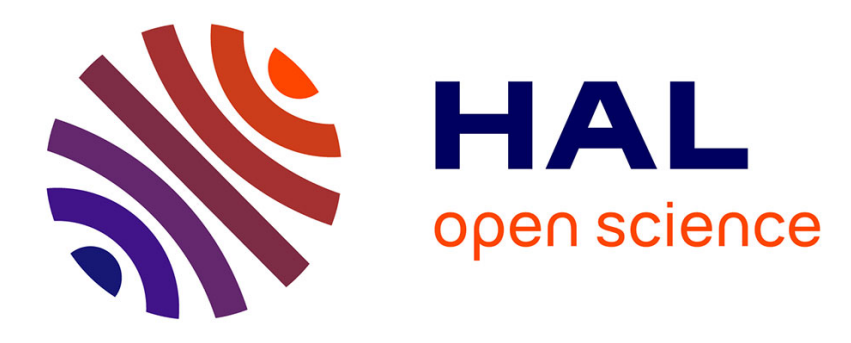

\title{
Étude des métaux avec le microscope ionique de champ
} M. Drechsler

\section{To cite this version:}

M. Drechsler. Étude des métaux avec le microscope ionique de champ. Revue de Physique Appliquée, 1968, 3 (4), pp.331-336. 10.1051/rphysap:0196800304033100 . jpa-00242868

\section{HAL Id: jpa-00242868 https://hal.science/jpa-00242868}

Submitted on 1 Jan 1968

HAL is a multi-disciplinary open access archive for the deposit and dissemination of scientific research documents, whether they are published or not. The documents may come from teaching and research institutions in France or abroad, or from public or private research centers.
L'archive ouverte pluridisciplinaire HAL, est destinée au dépôt et à la diffusion de documents scientifiques de niveau recherche, publiés ou non, émanant des établissements d'enseignement et de recherche français ou étrangers, des laboratoires publics ou privés. 


\title{
ÉTUDE DES MÉTAUX AVEG LE MIGROSGOPE IONIQUE DE GHAMP
}

\author{
Par M. DREGHSLER, \\ Laboratoire des Mécanismes de la Croissance Cristalline, associé au C.N.R.S., \\ Université d'Aix-Marseille, Faculté des Sciences Saint-Jérôme, Marseille, $13^{\text {e }}$.
}

\begin{abstract}
Résumé. - Les méthodes d'étude des métaux avec le microscope ionique de champ de E. W. Müller sont décrites. On observe des dislocations. On trouve individuellement des atomes étrangers et des lacunes. On peut voir toutes les faces limites entre cristaux sous forme de lignes à la surface. On observe la forme d'équilibre du cristal et on mesure les énergies de diffusion de surface. On peut analyser les atomes ou molécules à la surface, si on combine la pointe d'émission avec un spectromètre de masse. Les aspects positifs et négatifs de ces méthodes d'étude sont indiqués.
\end{abstract}

Abstract. - The methods of investigating metals with the field ion microscope of E. W. Müller are described. Dislocations are observed. Foreign atoms and vacancies are found individually. Different types of boundaries between crystals are seen as lines at the surface. The equilibrium shape of crystals is observed. Energies of surface diffusion are measured. Atoms or molecules at the surface are analysed, if the field emitter is combined with a mass spectrometer. Positive and negative aspects of these methods are indicated.

L'étude des métaux avec des microscopes ioniques de champ est un domaine de travail nouveau et intéressant. Ici, après une courte description du microscope, on signale quelques méthodes importantes; on donne des exemples typiques et on discute quelques perspectives. On a choisi de préférence des exemples de recherches conduites par les collaborateurs de l'auteur et par lui-même au Fritz-Haber-Institut de la Max-Planck-Gesellschaft à Berlin.

Le microscope ionique de champ a été construit en 1950 et développé jusqu'à aujourd'hui par E. W. Müller [1-3]. L'auteur était son assistant entre 1949 et 1952. L'objet étudié avec ce microscope est l'extrémité d'une petite pointe métallique, par exemple de tungstène, d'un diamètre d'environ $10^{3} \AA$. La pointe est produite habituellement à partir d'un fil étiré. Le fil est attaqué chimiquement pour former un cône très pointu. Une telle pointe est fixée dans le microscope en face d'un écran fluorescent ( fig. 1). Dans le microscope, on établit une pression partielle d'un gaz, en général $10^{-3}$ torr d'hélium. Si on donne à la pointe un potentiel de l'ordre de $15000 \mathrm{~V}$ par rapport à l'écran, il résulte au bout de la pointe un champ électrique qui atteint $10^{8}$ à $10^{9} \mathrm{~V} / \mathrm{cm}$, à cause des très petites dimensions de la pointe. Dans un champ aussi intense, la barrière de potentiel d'un atome d'hélium neutre est suffisamment déformée pour qu'un électron puisse s'échapper par effet tunnel et entrer dans le métal. Un ion d'hélium ainsi produit est accéléré vers l'écran. Là, la totalité des ions d'hélium produit une image très agrandie des emplacements à forte probabilité d'ionisation. Cette probabilité est particulièrement élevée devant les atomes qui forment de petites protubérances à la surface au bout de la pointe. Pour cette raison, l'image ionique donne la

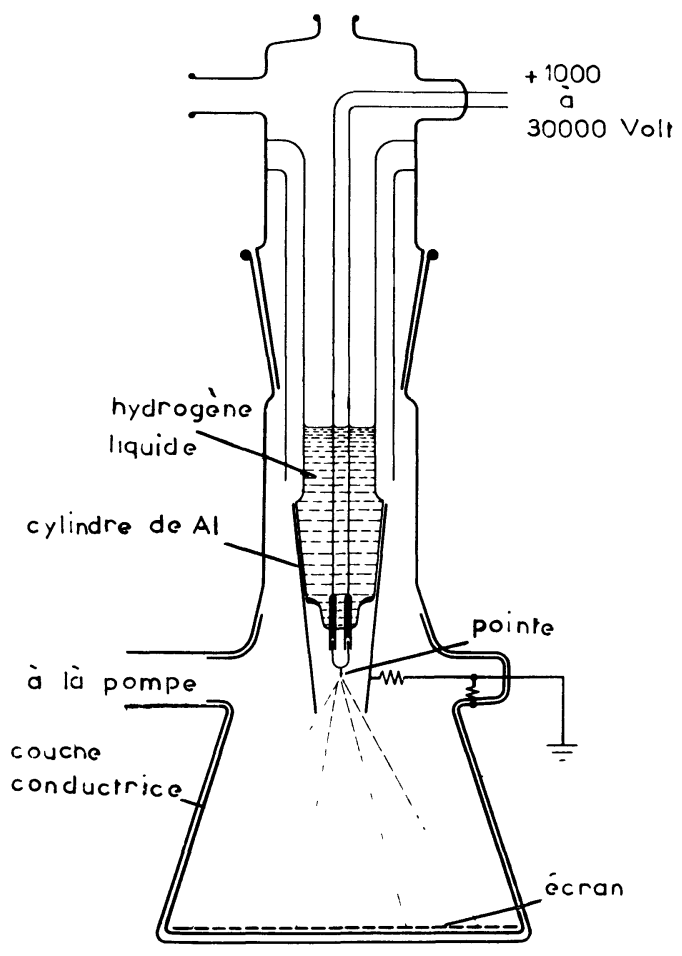

FIG. 1.

Microscope par émission de champ (Erwin W. Müller). 


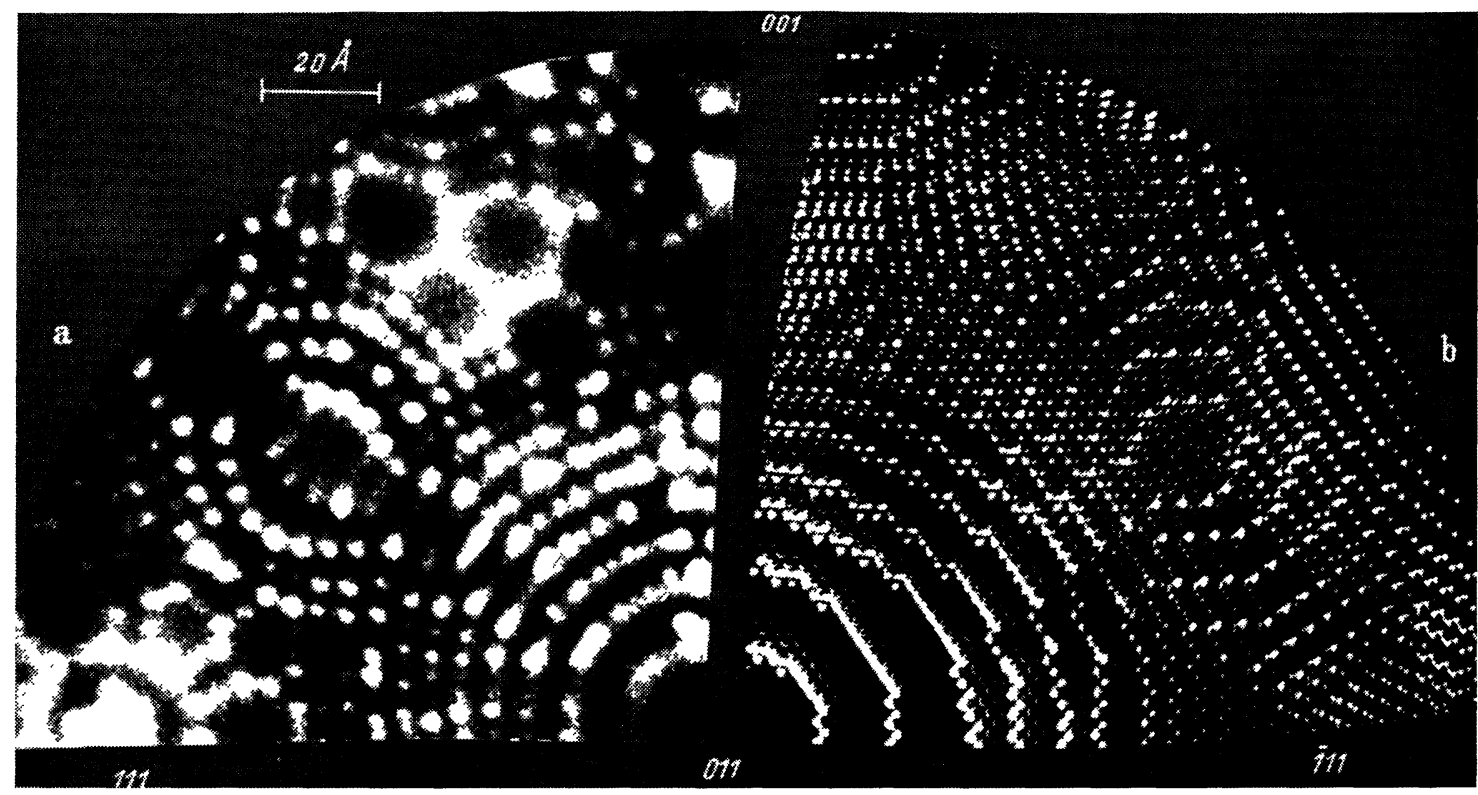

FIG. 2.

a) Section d'une image ionique de la surface d'une pointe de tungstène.

b) Photographie d'un modèle de cristal cubique centré avec une surface arrondie (M. Drechsler et P. Wolf).

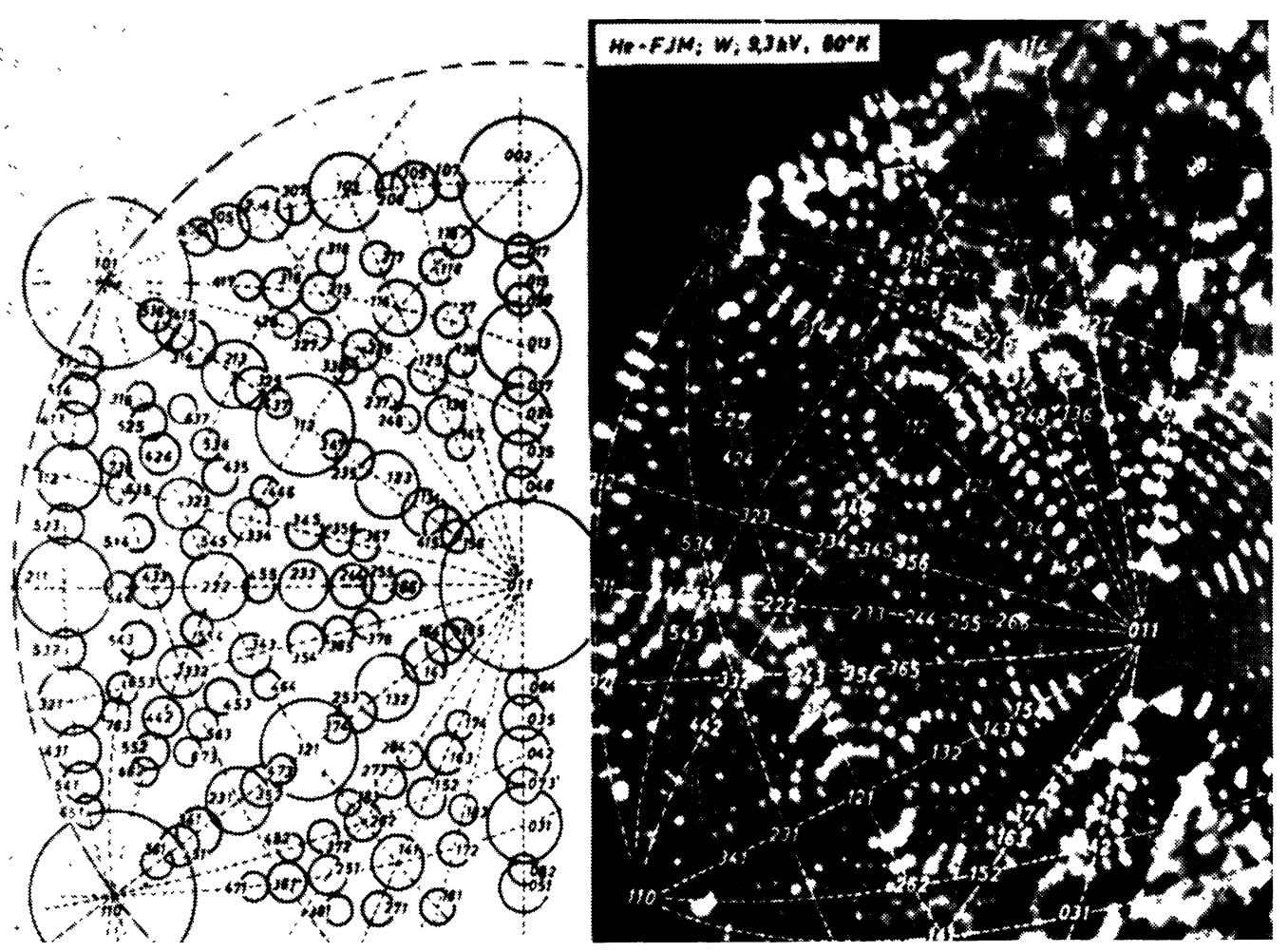

FIG. 3. - Projection d'indices de Miller et image ionique de $\mathrm{W}$ avec indices et lignes de zone (M. Drechsler et H. Liepack). 
position de ces atomes de la surface. On ne traite pas ici d'autres détails de la formation de l'image, comme le rôle du dispositif de refroidissement de la figure 1. Beaucoup de résultats obtenus avec ce microscope peuvent être compris sans savoir tout cela. Il suffit de savoir que les atomes de la surface cristalline à peu près hémisphérique sont reproduits dans une sorte de projection sur l'écran.

On doit se rappeler que, outre ce microscope ionique de champ, il existe aussi le microscope à électrons de champ [3]. La construction de ce microscope est très analogue, mais l'image n'est plus produite par des ions mais par des électrons de champ avec une polarité opposée de la pointe. Les deux microscopes se complètent. Quelquefois, on peut produire l'image de la surface aussi bien avec des ions qu'avec des électrons. Le microscope à électrons de champ convient surtout aux études d'adsorption, bien que les atomes individuels du réseau ne soient pas résolus.

La pointe métallique fabriquée par attaque chimique a une surface rugueuse et irrégulière qui n'est pas convenable pour la production d'une image de bonne résolution atomique. Pour cela, il faut produire une surface cristalline à peu près hémisphérique, qui contient des gradins atomiques, en appliquant un champ un peu supérieur au champ nécessaire pour obtenir l'image. Ce champ arrache successivement les atomes protubérants (évaporation de champ).

Si le cristal a la forme d'un hémisphère, un plan réticulaire coupe la surface selon un cercle qui peut être transformé en ellipse, par projection. Des plans réticulaires parallèles apparaissent, par conséquent, en projection, comme des cercles ou des ellipses concentriques. Puisqu'un cristal contient plus d'un groupe de plans réticulaires parallèles, il faut s'attendre que les atomes soient arrangés en plusieurs systèmes de cercles concentriques qui se superposent. C'est ce qui se passe, comme le montrent les figures 2 a [4] et suivantes. A titre de comparaison, la figure 2 montre la photographie d'un modèle de cristal dont la surface a une forme sphérique. Les points blancs de l'image qui forment un cercle sont des atomes en bordure d'un plan réticulaire. La surface à l'intérieur du plus petit cercle d'un système est en général une surface idéale. Sur les faces compactes, on ne voit que les atomes du bord, sauf pour quelques exceptions.

On trouve les indices des faces en comparant l'image avec une projection d'indices calculés (fig. 3) [5]. Comme on le voit, il y a aussi sur la surface produite par évaporation de champ beaucoup de faces d'indices élevés.

On ne peut pas obtenir une résolution atomique avec tous les métaux parce que le champ nécessaire pour produire l'image peut produire aussi l'évaporation de champ. Cependant, on a réussi à augmenter le nombre des métaux utilisables avec des procédés spéciaux dont on ne parlera pas ici. Il y a maintenant des résultats positifs avec $\mathrm{W}, \mathrm{Mo}, \mathrm{Pt}, \mathrm{Ta}, \mathrm{Co}, \mathrm{Re}, \mathrm{Ir}$, $\alpha-\mathrm{Fe}$, quelques autres métaux et quelques alliages.
On peut obtenir de telles images avec résolution des atomes du réseau depuis quatorze ans (E. W. Müller). La technique semble très simple ( $f g .1$ ), mais elle nécessite beaucoup de techniques accessoires et un apprentissage de plusieurs années pour obtenir des résultats de valeur.

Cette méthode a été appliquée dans diverses directions pour l'étude des métaux. Sur ce sujet, on présente quelques exemples typiques. La place manque pour traiter ici d'autres questions intéressantes qui touchent, par exemple, les dommages par irradiation, les whiskers et les changements de phases.

Dislocations. - Il y a douze ans environ que des dislocations ont été trouvées avec ce microscope [4]. Depuis, plusieurs laboratoires travaillent dans cette direction. La figure 4 montre l'image d'une disloca-

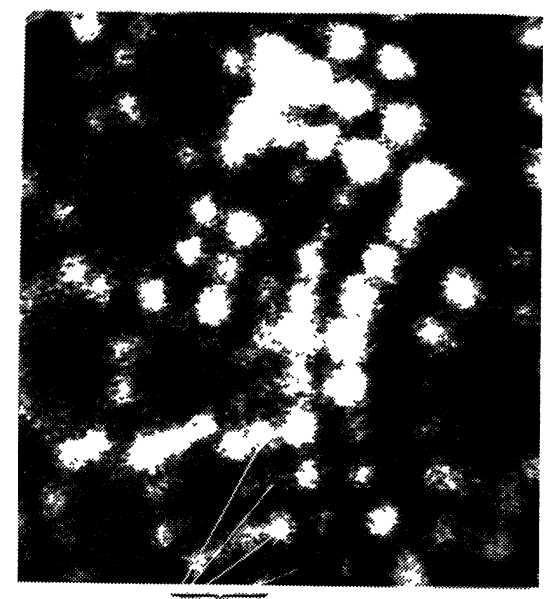

FIG. 4. - Dislocation. Section d'une image ionique. La chaîne d'atomes indiquée se termine au-dessus $\mathrm{du}$ centre de l'image (M. Drechsler et P. Wolf).

tion. On voit comment une chaîne d'atomes se termine. A cet endroit, une dislocation débouche à la surface. Une telle image n'indique pas la direction de la dislocation et le vecteur de Burgers. Cependant, on peut les trouver si on évapore le cristal par champ en observation continue. L'espoir d'étudier le cœur des dislocations n'a été réalisé que partiellement. On peut décider, par exemple, si une dislocation est une dislocation parfaite ou bien si elle est dissociée en deux dislocations partielles avec une distance de $50 \AA$ par exemple. Mais il n'est pas possible de déterminer la position exacte des atomes au cœur de la dislocation. La difficulté est que l'on ne peut pas mesurer avec précision la distance entre deux atomes voisins. En outre, les atomes au cœur de la dislocation sont un peu déplacés si ces atomes arrivent à la surface au cours de l'évaporation de champ. Une difficulté supplémentaire dans l'étude des dislocations est que le champ cause au cristal des contraintes mécaniques dépassant la limite de rupture technique, c'est-à-dire 
qu'il faut supposer que les dislocations observées sont modifiées par cette contrainte. Mais si, dans l'avenir, les recherches des dislocations avec ce microscope augmentent, il ne faut pas attendre que cette méthode prenne une importance semblable à celle qui utilise le microscope électronique classique.

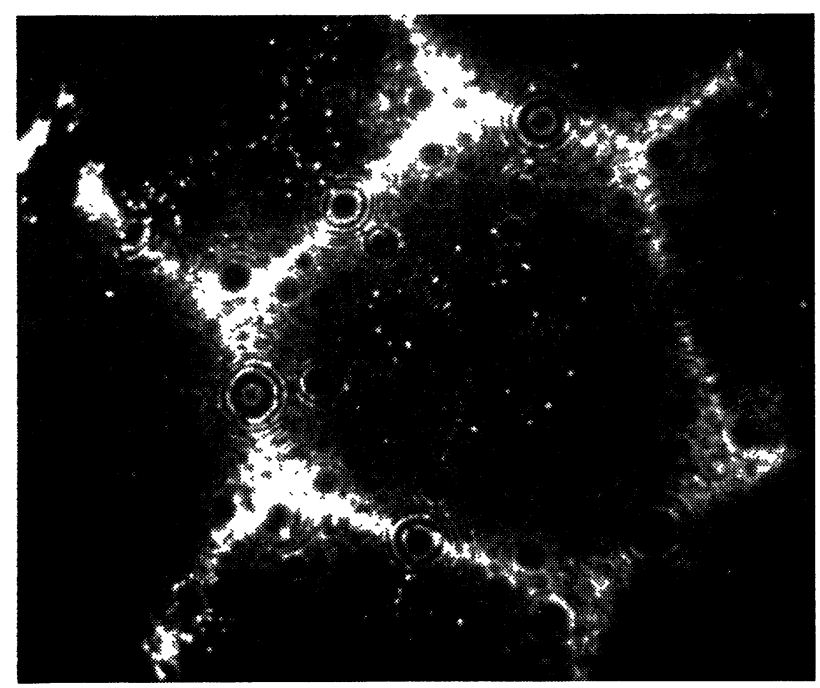

Fig. 5. - Molécules individuelles d'oxyde d'aluminium à la surface de l'iridium (G. Pankow et M. Drechsler).
Atomes étrangers et lacunes. - Avec ce microscope, on peut observer des atomes (molécules) étrangers et des lacunes. La figure 5 en montre un exemple [6]. Les points blancs brillants sont des molécules individuelles d'oxyde d'Al. Dans ce cas, les molécules sont évaporées sur la surface. Cependant, des atomes étrangers inclus dans le réseau sont observables si la surface les atteint au cours de l'évaporation de champ [7]. Malheureusement il y a une difficulté : c'est que les atomes sont en général désorbés par le champ formant l'image. Et dans les rares cas où les atomes étrangers restent sur la surface, les forces de liaisons sont modifiées par le champ. Bien que les atomes étrangers désorbent, il semble qu'ils causent souvent un déplacement d'un atome du réseau avant d'être désorbés et que cet atome déplacé devient visible ${ }^{(1)}$. Ainsi, on peut compter les atomes étrangers et déterminer leur position.

On peut également identifier des lacunes s'il manque des atomes dans l'image du cristal évaporé par champ.

(1) Récemment, cette hypothèse de l'auteur a été vérifiée par S. Brenner, U.S. Steel Corp., Monroeville Pa. Les mesures effectuées par M. Brenner à l'aide d'un microscope-analyseur d'atomes ont montré que les points brillants qu'on observe sur l'écran après l'arrivée de quelques atomes d'azote à la surface d'une pointe de tungstène ne sont pas des atomes d'azote, mais des atomes de tungstène (15th Field Emission Symposium, Bonn, septembre 1968).

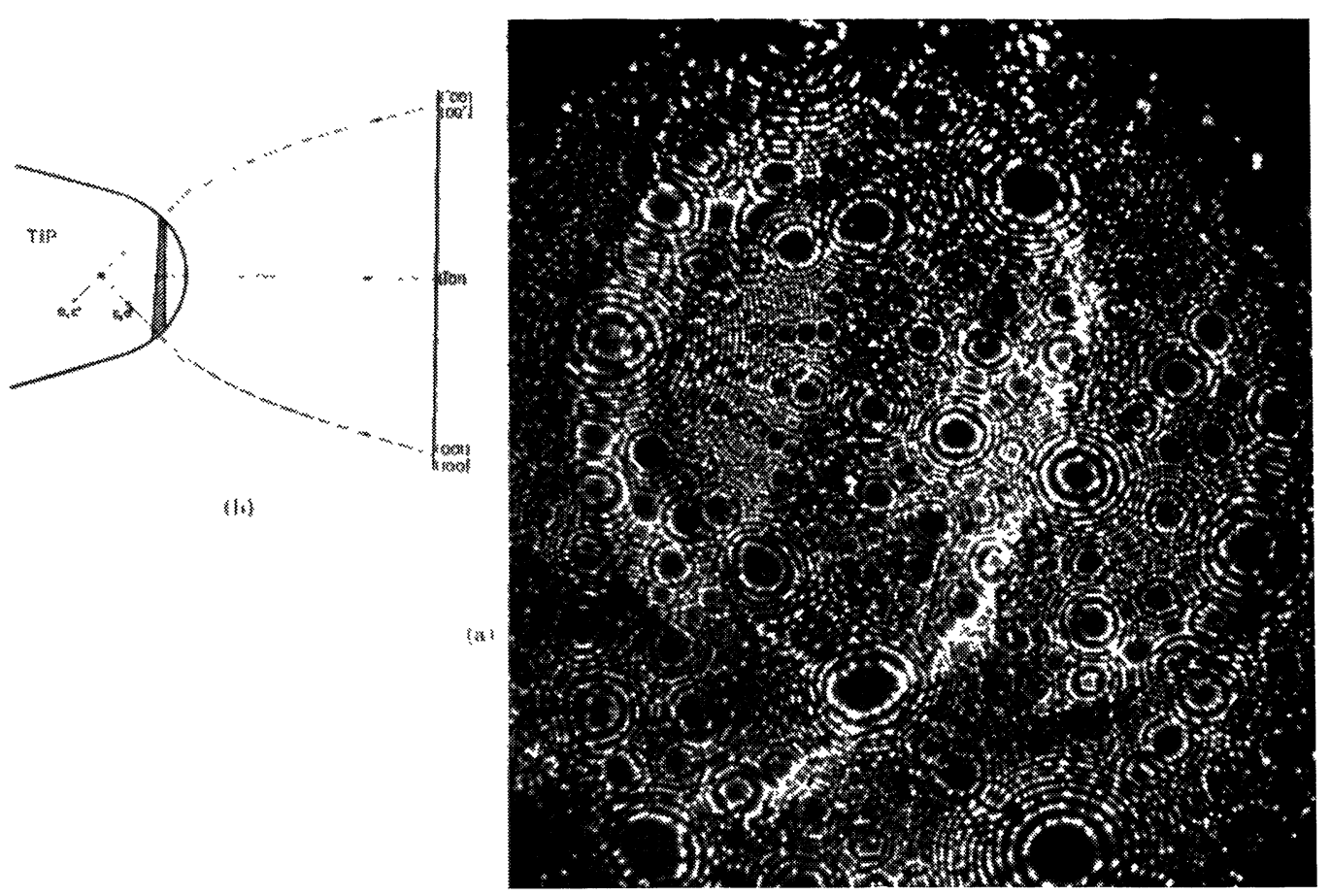

FIG. 6.

(a) Alliage ordonné Pt-Co $(50 \%)$ avec une couche de $20-80$ Å d'épaisseur et $90^{\circ}$ de différence d'orientation.

(b) Position de la couche (T. T. Tsong et E. W. Müller). 
Il y a cependant des difficultés dues aux phénomènes suivants : 1) Les lacunes de la surface peuvent être restaurées par déplacements des atomes dans le champ; 2) Inversement, le champ peut produire des lacunes à la surface. Ces deux phénomènes ne sont pas encore bien étudiés.

Cependant, on peut admettre que les recherches sur les atomes étrangers et les lacunes avec ce microscope semblent très prometteuses (voir aussi le paragraphe : Microscope-analyseur d'atomes de la surface).

Faces limites dans les métaux. - On peut observer, avec ce microscope, des faces limites dans les métaux, comme joints de grains, joints de domaines, joints de macles et fautes d'empilement. La figure 6 en donne un exemple [8]. C'est un alliage ordonné. Mais dans les alliages désordonnés on ne trouve qu'une répartition plus ou moins statistique des points dans l'image. Le cristal représenté par la figure 6 contient une couche d'épaisseur de 20-80 $\AA$ qui a une orientation de $90^{\circ}$ par rapport au cristal. Les deux faces limites apparaissent dans l'image comme deux grands cercles un peu sombres. On voit particulièrement bien entre le centre de l'image et le bord gauche de l'image les deux faces limites et les différentes orientations de la couche.

On doit s'attendre à voir cette étude des faces limites et des orientations devenir un domaine d'étude intéressant.

Structure des surfaces et croissance cristalline. On peut de même mettre en évidence avec ce microscope des structures de surface et des formes cristallines qui ne sont pas influencées par le champ. La figure 7 montre un exemple. Le cristal de tungstène représenté a été d'abord chauffé à $2200^{\circ} \mathrm{K}$. La forme d'équilibre du cristal est ainsi produite et elle se compose de faces planes (cercles sombres de la figure 7) et de régions arrondies. Une forme d'équilibre des monocristaux métalliques qui n'est pas influencée par des impuretés était mesurée jusqu'à ce jour seulement avec des microscopes à émission de champ [5], [9], [10] et on a trouvé un accord quantitatif avec les formes calculées. Sous l'influence de l'adsorption, une autre forme d'équilibre est produite ( $f i g .7 \mathrm{~b}$ ). En outre, la figure 7 montre que, sur les faces d'indices élevés, existe un désordre thermique.

On peut donc avec ce microscope faire des études fondamentales de la croissance cristalline. A Marseille, l'auteur étudie maintenant l'influence des couches d'adsorption définies sur la forme cristalline et ainsi on peut espérer pouvoir mesurer les énergies de liaison des atomes étrangers sur certaines faces d'un monocristal.

Diffusion de surface. - Pour mesurer l'énergie de diffusion d'un atome sur une face définie, on évapore des atomes (molécules) sur la surface propre d'une pointe dans le microscope. Ainsi on obtient des images des atomes individuels adsorbés, comme sur la figure 5, ou sur la figure $7 \mathrm{~b}$ l'analogue des deux points sur la face centrale. Le temps moyen avant le déplacement est mesuré en fonction de la température et ainsi l'énergie de déplacement est mesurée avec un diagramme d'Arrhénius. Par exemple, pour l'autodiffusion de surface, la mesure de cette énergie pour les différentes faces d'un monocristal a été faite seulement avec des microscopes à émission de champ [11], [12], [13].

Microscope-analyseur d'atomes de la surface. On peut analyser les molécules ou les atomes à la surface d'une pointe cristalline si ces particules sont

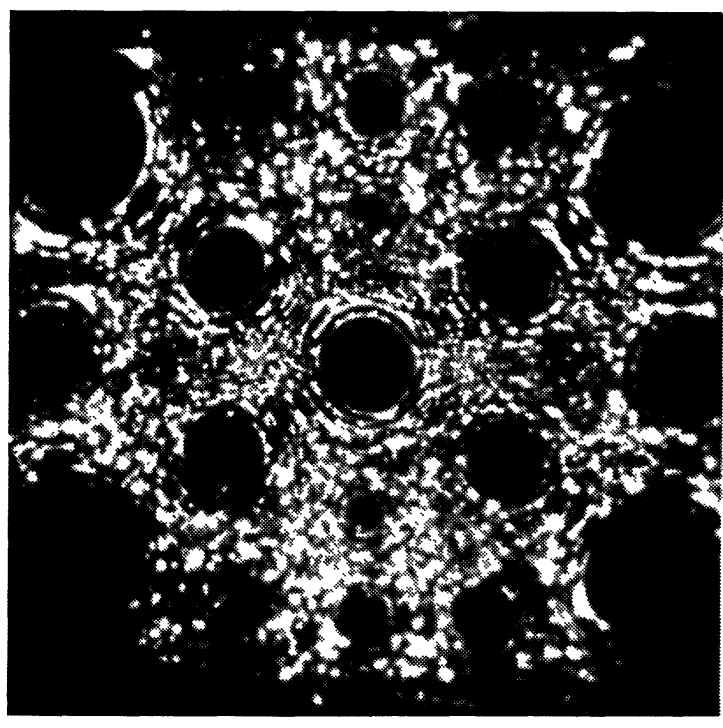

a

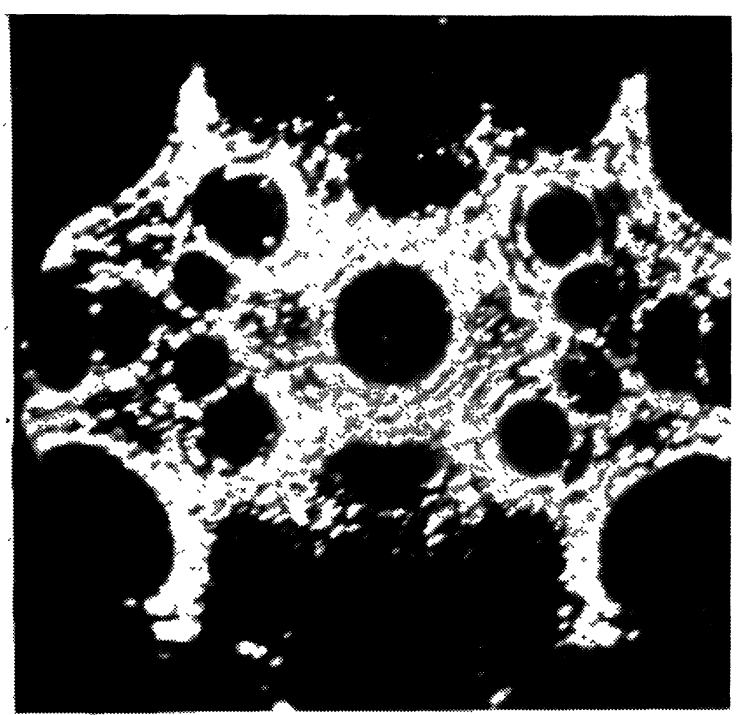

$\mathrm{b}$

FIG. 7. - Structure de surface et forme du cristal après chauffage (sans évaporation de champ) :

a) $2200 \circ \mathrm{K}$, sans adsorption.

b) Même cristal mais ayant changé de forme sous l'influence de l'adsorption de carbone (A. Müller et M. Drechsler) [10]. 
désorbées et ionisées par le champ et ensuite dirigées dans un spectromètre de masse. De tels appareils (spectromètres de masse à ions de champ) sont produits industriellement par la firme Varian (U.S.A. et R.F.A.) d'après un brevet de l'auteur.

Récemment, E. W. Müller [14] a réussi à mesurer la masse des atomes individuels avec une combinaison d'un spectromètre de masse à temps de vol et à ions de champ et un microscope à ions de champ (Atomprobe field ion microscope). On choisit sur l'écran du microscope l'image d'un atome qui doit être analysé. On déplace cette image jusqu'à ce que l'image de l'atome choisi vienne coïncider avec un petit trou percé dans l'écran. L'atome choisi est désorbé par champ par une impulsion de tension; il franchit le trou et est enregistré par un détecteur d'ions à multiplication d'électrons secondaires. La masse de l'atome est mesurée par son temps de vol qui est déterminé en mesurant la différence de temps entre l'impulsion de tension et l'impulsion du multiplicateur.

La possibilité d'analyser des atomes et molécules individuels situés à la surface (et peut-être aussi à l'intérieur) conduira certainement à plusieurs applications intéressantes.

Comparaison avec d'autres méthodes de recherche. - La recherche des métaux avec ce microscope diffère des autres méthodes (rayons X, électrons, neutrons, etc.) par les aspects positifs et négatifs qui suivent :

Aspects positifs :

1) C'est le seul instrument avec lequel on peut résoudre individuellement des atomes;

2) G'est un instrument avec lequel presque tous les types de défauts cristallins et les phénomènes de surface peuvent être observés;

3) On peut déterminer la nature des atomes sur la surface par spectrométrie de masse;

4) On peut étudier des phénomènes sur chacune des nombreuses faces du monocristal;

5) Les recherches peuvent être conduites dans l'ultravide bien que, pour former l'image, il faille introduire un gaz comme l'hélium ( $\simeq 10^{-3}$ torr);

6) Bien que seule la surface du cristal soit visible, on peut obtenir des informations sur l'intérieur du cristal en enlevant les couches successivement par évaporation de champ.

\section{Aspects négatifs :}

1) On ne peut étudier que des échantillons en forme de fil; il y a des exceptions, par exemple les whiskers;

2) Les cristaux étudiés sont très petits; leur diamètre est en général $<0,3 \mu$;

3) On ne voit que la surface du cristal et seulement à peu près $20 \%$ des atomes de la surface : ceux qui forment saillie;

4) On détruit le cristal quand on l'évapore pour en étudier le volume;

5) Normalement, on ne voit que la surface produite par l'évaporation de champ. Cependant, il y a des exceptions ( fig. 7);

6) On voit seulement une face soumise à un champ de $10^{8}$ à $10^{9} \mathrm{~V} / \mathrm{cm}$. Les conséquences sont :

- pour quelques métaux, le champ produit une évaporation de champ considérable, c'est-à-dire que le cristal est détruit et non visible,

- presque tous les atomes étrangers sont désorbés, seuls ceux qui restent peuvent être étudiés,

- l'énergie et les forces à la surface sont fortement modifiées par le champ. Cependant, on peut faire des expériences en champ nul, tremper le cristal et observer à basse température (voir par exemple le paragraphe : Diffusion de surface),

- le cristal observé est soumis à de très grandes contraintes mécaniques (voir le paragraphe : Dislocations).

7) Toutes les mesures des distances même entre atomes voisins contiennent une erreur considérable;

8) L'image n'est visible qu'avec des yeux habitués à l'obscurité et sa photographie exige des temps de pose considérables. Cependant, des intensificateurs d'image peuvent être utilisés;

9) Il faut former l'image à basse température $(<100 \mathrm{o})$, si on ne veut pas perdre le pouvoir séparateur.

Compte tenu de ces aspects, la recherche sur les métaux avec ce type de microscope est indispensable pour résoudre certaines questions fondamentales.

\section{BIBLIOGRAPHIE (sommaive)}

[1] MÜLlER (E. W.), Z. Physik, 1951, 131, 136.

[2] MÜLLER (E. W.), Adv. in Electronics, 1960, XIII, 83. MÜLLER (E. W.), Science, 1965, 149, 591.

MÜLLER (E. W.), Abh. Deutsche Akad. d. Wiss., Kl. Math. Phys. und Technik, 1967, 107.

[3] Good (R. H.) et MüLIER (E. W.), Handb. d. Physik, 1956, XXI, 176.

[4] DREChSLER (M.) et WolF (P.), IV. Int. Kongr. Elek. Mikr. 1958, Berlin, 1960, V, 1, 835.

DRECHSLER (M.), Z. Metallk., 1956, 47, 305.

MÜLLER (E. W.), Int. Kongr. Elek. Mikr. 1958, Verh. I, 1960, 835.

[5] DREChSLER (M.) et LIEPACK, Coll. Int. du C.N.R.S., Paris, 1965, 152, 49.
[6] Pankow (G.) et Drechsler (M.), Z. Physik. Chemie N.F., 1962, 31, 288.

[7] MÜLLER (E. W.), Z. Physik, 1959, 156, 399.

[8] Tsong (T. T.) et Müliter (E. W.), J. Appl. Physics, 1967, 38, 545

[9] DRECHSLER (M.) et Nicholas (J.), J. Phys. Chem. Solids, 1967, 28, 2609.

[10] DRECHSLER (M.) et MÜLLER (A.), J. Cryst. Growth, 1968, 3, 518 ; MÜLILER (A.) et DRECHSLER (M.), Surface Science, 1968/69, sous presse.

[11] EHRLich (G.) et HudDA (F. G.), J. Chem. Phys., $1966,44,1639$.

[12] MÜLLER (E. W.), Z. Physik, 1949, 126, 642.

[13] DREChSLER (M.) et VANSELOW (R.), Z. Kristallogr., 1956, 107, 161.

[14] MÜLLER (E. W.), Rev. Scient. Inst., 1968, 39, 83. 